\title{
Corela
}

Cognition, représentation, langage

HS-8 | 2010

L'interpellation

\section{L'interpellation entre immobilisation et mise en mouvement. Un genre dialogique et situé de l'activité}

Jean-Paul Bernié

\section{(2) OpenEdition}

Journals

Édition électronique

URL : http://journals.openedition.org/corela/732

DOI : $10.4000 /$ corela.732

ISSN : 1638-573X

Éditeur

Cercle linguistique du Centre et de l'Ouest - CerLICO

Référence électronique

Jean-Paul Bernié, « L'interpellation entre immobilisation et mise en mouvement. Un genre dialogique et situé de l'activité », Corela [En ligne], HS-8 | 2010, mis en ligne le 24 novembre 2010, consulté le 19 avril 2019. URL : http://journals.openedition.org/corela/732 ; DOI : 10.4000/corela.732

Ce document a été généré automatiquement le 19 avril 2019

\section{(c) (i) (2)(2)}

Corela - cognition, représentation, langage est mis à disposition selon les termes de la licence Creative Commons Attribution - Pas d'Utilisation Commerciale - Partage dans les Mêmes Conditions 4.0 International. 


\title{
L'interpellation entre immobilisation et mise en mouvement. Un genre dialogique et situé de l'activité
}

\author{
Jean-Paul Bernié
}

1 Suffit-il qu'il y ait, de la part d'un locuteur quelconque, intention d'interpeller, pour que l'on puisse parler d'interpellation? Rien n'est moins sûr. L'interpellation sera vue ici sous l'angle de celui sans lequel elle n'existe tout simplement pas : l'interpellé. Si l'énonciation de l'interpelleur n'a aucune unité linguistique, ce n'est pas un hasard : l'interpellation est une action co-construite dans des situations dialogiques ; seuls ses effets disent sa nature. D'où la fragilité des différenciations formelles entre apostrophe, intimation, assignation, ou interpellation. Une conception réellement dialogique de l'énonciation suppose que la forme sémiotique de l'interpellation soit cherchée, non pas dans l'hypostase de l'action d'un locuteur solipsiste, mais dans une approche holistique des situations, englobant l'ensemble des partenaires et l'objet de leur interaction. Les trois " All hail, Macbeth!» des sorcières de Shakespeare ${ }^{1}$ doivent-ils être considérés comme un "grand salut " (traduction ordinaire) ou comme une interpellation? Ce sont les effets produits sur le héros qui le diront: si Macbeth se contente de répondre : « Hail to thee, you witches!", et de passer son chemin, il y aura eu salut mais pas interpellation, quelle qu'ait pu être l'intention des sorcières ou l'origine de l'attitude de Macbeth. S'il y a eu interpellation, ce n'est pas non plus parce que " to hail » est le même verbe que notre " héler ", supposé interpellatif, c'est parce que Macbeth tuera Duncan. Lorsqu'il rencontre les sorcières, Macbeth est Comte de Glamys et il le sait ; il est aussi, de fait, comte de Cawdor, mais il ne le sait pas encore; c'est lorsqu'il le saura qu'il pourra accorder créance au dernier « salut ", qui fait de lui le roi, et qu'il pourra donc se mettre en mouvement vers un destin nouveau. Cette prise d'identité est l'objet réel de la situation ; c'est le partage de cet objet dialogique qui permet de parler d'interpellation. D'où un possible balisage provisoire de la notion autour des quelques repères suivants, distinguant l'interpellation des notions voisines : 
2 1. Elle joue sur une contradiction de dicto ou de re entre ce qui est et devrait / pourrait être. Il y a là une propriété de toute exclamation (Martin, 1987) et de la position psycholangagière qu'elle suppose (Grize \& Piérault-Le Bonniec, 1983).

3 2. Elle est adressée, mais dans une situation où le récipiendaire perçoit un enjeu d'identité. Macbeth est interpellé parce qu'il entrevoit une autre manière de se penser lui-même à travers l'organisation de son rapport à autrui.

4 3. Elle est pour le récipiendaire ouverture de sens, projection au delà du préconstruit : pour Macbeth, la situation, voire sa vie, vont pouvoir signifier quelque chose, mais en laissant plusieurs chemins ouverts, à arpenter.

5 4. Elle suppose un arrière-plan évaluatif. C'est à partir de la posture "d'arpenteur » cidessus (Jorro, 2001), que va se définir l'identité nouvelle. L'énonciation interpelle lorsqu'elle est perçue comme réévaluant un contexte pour le transformer.

6 L'apprentissage dans une institution reproductrice d'idéologie rend pertinent le schéma pragmatique ci-dessus, même dans une situation banale de rappel à l'ordre d'un cancre par un professeur: accepter ou rejeter sa remarque constitue une étape de la subjectivation. L'interpellation pointe l'effet produit sur un sujet, parfois collectif, par des configurations discursives hétéroclites prenant contextuellement pour le destinataire une dimension perlocutoire fruit d'une autorité dotée du pouvoir d'évaluer, plaçant le sujet à la croisée de chemins divergents, ouvrant une crise -au sens étymologique- entre des exigences contradictoires qu'il s'agisse de faits ou de valeurs. En effet, rien a priori ne voue ces configurations à placer l'allocutaire davantage en garde à vue que sur son chemin de Damas.

\section{Il est donc indispensable de s'interroger sur la portée réelle de la bifurcation entre fixation et déplacement.}

7 L'effet de l'interpellation, par un professeur ou un policier, se réduit-il à une forme ou une autre d'assujettissement, avec un faible degré d'ouverture dépendant étroitement de la subjectivité du sujet? Il n'est pas contestable que l'interpellation ait quelque chose à voir avec ce que J. Butler (1997) appelle «the psychic life of power », titre d'un ouvrage sous-titré «theories in subjection»: autant assujettissement que subjectivation (est-ce un hasard ?). Dans le cas le plus banal, celui de l'interpellation pour assurer l'ordre de la classe, par exemple en direction d'un élève agité ( Eh, Untel, tu crois que c'est comme ça qu'on se comporte en cours de morale?»), la marge du récipiendaire est faible : il peut répondre : «Mais Monsieur, je n'ai rien fait ». Cette identité peut se révéler injouable par impossibilité d'enrayer le moteur du dialogisme, à savoir: la garantie pour le fautif d'accéder à une forme de reconnaissance dans le changement exigé. L'on peut donc suivre un moment J. Butler lorsque, reprenant la théorie de l'interpellation d'Althusser, elle reformule l'effet produit par exemple sur un passant dans la rue : «il marche en regardant devant lui et en pensant à ses propres affaires; une voix, dans son dos, le siffle impérieusement :

8 " “Vous, là-bas!"; aussitôt, sans même prendre le temps de la réflexion, il se retourne, peut-être parce qu'il est incité à le faire par un sentiment de culpabilité latent, (...) qui lui fait instinctivement interpréter l'appel comme une injonction à caractère légal venue 
d'un gardien de l'ordre auquel il a, comme on dit, à "'répondre" de lui-même et de ses actes. (...) L'individu qu'était le passant, du fait de s'être senti convoqué par l'appel, et d'y avoir répondu en se retournant, sans avoir même un seul mot à prononcer, est devenu et s'est à la fois reconnu et fait reconnaître comme "sujet" en prenant place, "sa" place, dans l'ordre symbolique marqué par la loi et signifié par la force de la voix à laquelle il ne peut faire autrement que faire face ».

C'est sur cette vision qu'Althusser bâtit son explication du devenir-sujet de l'individu, qui consiste pour lui à trouver sa "juste" position, celle qui lui est assignée dans la relation de pouvoir imposée par l'intermédiaire du langage et représentée par la voix lançant l'injonction. L'idéologie constitue ses sujets en «hélant » des individus concrets : "All hail, children! that shalt be pupils hereafter! ». Ainsi se trouvent souvent réunis, dans l'ordinaire des situations de classe, les quatre facteurs qui font la «vie psychique du pouvoir » :

- le processus verbal d'interpellation qui saisit des individus comme sujets d'un contexte...

-...et qui pointe par là-même un assujettissement à une sorte de Sujet transcendant : la voix de la Loi.

- la reconnaissance universelle des sujets dans ce Sujet, ressort.du dialogisme

- la garantie absolue que tout est bien ainsi : pour un sujet qui reconnaît ce qu'il est, tout ira bien.

10 La garantie se présente comme dotée d'un pouvoir d'immobilisation : elle fixe l'élève et son langage autour de quelques repères nominaux aux valeurs convenues, devenant obligées. A l'échelle des apprentissages, l'on voit ainsi se construire les comportements scolaires consuméristes. Ce type d'interpellation enfermera souvent l'élève dans un système de représentations de l'école réduite à la distribution de l'utilitaire, mais...

11 Exemple : des élèves de CE2 ont relevé des mots-clés en visionnant une émission portant sur leur thème d'étude en cours. Ils doivent ensuite écrire un résumé de l'émission, en réutilisant le plus grand nombre possible d'entre eux. Le maître lit le résumé d'une élève : «Tu n'as pas été assez précise dans ce résumé ! (il prend le cahier où figuraient les motsclés, l'ouvre et le montre à l'élève) Regarde, regarde, regarde ! Tout ça! Et rien n'apparait dans ton résumé alors qu'il y avait plein de choses intéressantes! » (l'élève et le maître se regardent silencieusement un instant, puis le maître se tourne vers un autre élève, et le premier remet pensivement son crayon dans sa bouche...).

13 Remarque : il faut signaler le rôle d'un élément trouvant sa place dans une linguistique de l'activité : le plan intonatif. Comment le maître dit-il « Regarde, regarde, regarde » ?²

Ici, en l'absence d'indications intonatives, il semble impossible de trancher entre dimensions fermante et ouvrante, immobilisation et déplacement. Soit le maitre se réfère à un ensemble de normes établies, de contrats antérieurs, concernant par exemple la réussite d'une activité résumante, et l'on pourrait alors conclure à un rappel conventionnel et normatif. Mais la répétition de «regarde » semble vouloir impliquer personnellement l'élève dans cette évaluation pour l'amener à une exploitation plus poussée de ses propres possibilités. Fermeture, au vu du rappel implicite de la norme du résumé scolaire français, ou ouverture de sens, l'élève étant sollicité pour prendre en main ses propres capacités? Question indécidable au vu des critères de l'analyse linguistique traditionnelle, qui peine à sortir d'une tradition de modélisation exclusive de l'écrit phrastique. 

ifurcations, instrumentées par l'interpellation : ce que nous appelons une « communauté discursive X-scolaire » (Bernié, 2002) en construction, en référence à la fois aux communautés d'origine des savoirs transmis, à la nature scolaire de leur transposition, et à leur structuration disciplinaire, représentée par la variable « X ». 


\section{Que se passe-t-il donc lorsque d'immobilisante, l'interpellation semble devenir créatrice de mouvement?} l'idéologie peut-il se retourner à son tour, changer complètement de valeur? Peut-il basculer d'une entrée forcée dans ce qu'en termes lacaniens on appellerait le «symbolique " (l'univers des identités prédéfinies) vers un horizon libérateur? Pour cette école de pensée, il n'y aurait là qu'illusion, l'autre voie n'étant que fuite hors du réel. Si le cancre qui obtempère ou le passant qui se retourne pour répondre à l'injonction de la loi effectue bien son entrée dans le "symbolique ", en revanche l'élève qui, lui aussi se retourne, mais pour contourner l'obstacle, dénie la portée pragmatique de l'interpellation et loge sa liberté dans ce retournement du retournement... illusoire dans l'esprit de la théorie Althussérienne : dans ce cadre -et cela n'étonnera guère- on ignore la relation entre ce qui se passe dans la tête du sujet-assujetti, et la dynamique des situations dans lesquelles il se trouve, les possibilités de reconstruction psychique qu'elles offrent aux sujets. L'une des raisons de ne pas en rester à l'acte linguistique d'interpellation, au geste de retournement, est que le même acte, le même geste, peut engendrer immobilisation ou déplacement. L'interpellation est à double tranchant dans sa capacité à appeler des sujets à exister. Interpeller a longtemps signifié «appeler en interrompant»; pour qu'il y ait interpellation, il faut qu'il y ait appel mais la rupture a ses vertus mystérieuses, et son initiateur ne peut jamais être maitre de ses effets: les contextes sont plastiques. Le rapport entre l'instance évaluative et interpellative, et l'action sensée du sujet, est à la fois étroit et étranger à toute relation de causalité mécanique. Le nœud de l'interpellation est de susciter une auto-évaluation de l'apprenant, de mettre en mouvement la manière dont il comprend ses propres processus de réaction aux consignes et tâches, ou celle dont il interprète sa propre altérité. Nous nous trouvons là au cœur d'une polyopération que nous avons appelée " fictionnalisation »(Bernié, 1998), en adaptant un terme du Père Ong, Jésuite et psychologue cognitif américain (1982). L'ouverture d'un sens dépasse d'assez loin la question du sens de la consigne : le maitre qui déplore la pauvreté du résumé de l'élève ne vise peut-être que la norme française en matière de résumé; l'élève, lui en tirera peut-être la conséquence que le maître va voir de quel bois il se chauffe... si le contexte intersubjectif créé l'amène à se représenter l'instance magistrale sous les espèces de «l'ami critique » (Jorro, 2002), -celui qui permet d'être «soi-même avec les autres ».

Le versant sur lequel l'interpellation met en mouvement au lieu d'immobiliser n'est donc pas celui d'une subjectivité pure, mais celle d'un sujet mû par le contexte dans lequel se déroule son activité, et qu'il doit pouvoir sémiotiser. L'élève s'organise cognitivement et psychiquement en fonction d'une fictionnalisation qui consiste à attribuer une valeur à des ensembles de paramètres qui caractérisent le contexte de l'activité. Il peut être soigneusement aménagé, l'activité de l'élève n'en sera jamais une «application » au sens mathématique. Nos recherches sur ces questions nous amènent à poser trois nouvelles hypothèses, découlant de la primauté des savoirs en jeu, susceptibles de rendre compte du caractère potentiel d'ouverture du sens qui peut être celui de l'interpellation : 
27 a) le rôle du degré de stabilité ou d'instabilité du contexte intra-psychique reconstruit par l'élève (fictionnalisation). Le contexte y est vu comme l'affectation de valeurs aux paramètres de toute situation communicative (énonciateur, but, destinataire, lieu social). L'image d'énonciateur que l'élève se construit du maître, croisée avec celle du lieu social « école », peuvent entrer en synergie ou en contradiction, et varier d'un moment à l'autre dans une même situation -ce qui affecte plus encore, comme l'ont montré mes recherches sur les genres scolaires, ses réponses à la question : « en tant que qui est-ce que je parle / écris ?", donnant lieu à des postures tranchées (Rebière, 2001). Le contexte ne ressemble pas à un jeu de poupées russes; il n'y a pas de hiérarchie stable d'éléments, les uns centraux et les autres périphériques, mais un jeu fond / figures dont la position est toujours mouvante (Bernié, 2001; Grossen, 2001). Il y a là un facteur qui recoupe partiellement ceux que nous venons d'évoquer. D'où la labilité du sens de l'interpellation.

b) le stade de la topogenèse. Par ce terme, que nous travaillons au sein de l'ARCD ${ }^{3}$, nous désignons le transfert à l'élève de la responsabilité de la tâche par la construction d'une position d'énonciateur pertinent (Jaubert, 2007) qui lui permette d'assimiler en profondeur les savoirs scolaires, donc les manières d'agir-penser-parler cohérentes avec les configurations épistémiques en jeu (Bernié, 2002). Il est significatif que les interpellations « ouvrantes » interviennent dans ce que nous appelons, avec nos collègues de Montpellier, les situations intermédiaires, et que F. Ruellan (1999. Cf. Bernié, 2005) appelait " situations différées " : celles où se produit à la fois le choc entre connaissances et représentations anciennes et nouvelles, complexes et élaborées, et la subjectivation.

c) élément de la topogenèse, le statut de la garantie donnée dans les situations d'apprentissage à travers les règles du jeu: accepter le jeu de l'interpellant est une garantie de reconnaissance et de sécurité. Même le voleur inerpellé est certain d'avoir à y gagner : on lui saura gré d'avoir accepté d'être reconnu comme voleur. Mais en situation d'apprentissage, la teneur et le contexte de l'interpellation peuvent donner l'idée d'une garantie absolue de reconnaissance par conformation à la Loi, ou initier une posture de travail où il s'agisse de s'essayer soi-même. Il y a là une condition du retournement du retournement.

Bien entendu, ces trois facteurs dépendent de la situation mise en scène par l'enseignant, et celle-ci est fonction de la nature des savoirs en jeu, mais il serait naif de penser que cette, dernière soit dotée a priori d'une sorte d'épistémologie naturelle ou d'un quelconque déterminisme : c'est la relation entre interactions et construction de l'objet de savoir qui fait sens et alimente la fictionnalisation, toujours en mouvement. Il n'y suffit ni d'une ingénierie sophistiquée ni d'actes isolés. Deux exemples, pris dans deux champs de savoir spécifiques et nettement différents :

\subsection{Deux « courses à 20 » en classe de mathématiques au CE2. (ANNEXE 1)}

31 Dans l'une de deux situations rigoureusement identiques sur le plan des objectifs didactiques et de la macro-structure, aucune interpellation; l'autre en fourmille. Cette différence est en relation directe avec la nature des savoirs construits.

32 La " course à 20 » est une situation connue des didacticiens des mathématiques : deux élèves jouent à savoir lequel des deux pourra dire « 20 » le premier. L'on part de 0 . Le 
premier propose un chiffre, soit 1 soit 2 ; le second de même, et sa proposition est additionnée à celle du premier, qui enchaîne en ajoutant 1 ou 2 au total, et ainsi de suite : celui qui arrive à 20 le premier a gagné. Suivant la théorie des situations mathématiques, le jeu comporte plusieurs phases: action - formulation (les élèves discutent de leur stratégie) - institutionnalisation.

\subsubsection{Connivence immobilisante et absence d'interpellation.} caractérisée par le souci d'extraire des interventions des élèves quelque chose qui ressemble à la liste gagnante, qu'elle cherche à faire formuler. Elle ne comporte aucune interpellation.

34 A un stade médian du déroulement de la séance, les élèves ont explicité certains "théorèmes". Exemple: "Si on joue 14 on gagne". Une différenciation se fait jour, néanmoins, entre :

35

*ceux qui cherchent, vu leur argumentation, à se placer dans une perspective logicomathématique, en s'efforçant de distinguer le contingent, lié à l'action, du nécessaire, touchant aux conditions générales de réussite du jeu, mais sans parvenir à avancer réellement dans cette distinction. Ainsi :

-1012 , So. : mais c'est pas vrai parce que onze c'est/ on gagne pas toujours parce que là heu/ on avait mis heu/ treize quinze et dix-sept pis on a/ pis j'ai quand même/ puis/ Alison elle a quand même gagné

*ceux qui en restent à une position de jeu non distanciée ("j'ai joué 13") ou y reviennent avec des verbalisations non régulées par un raisonnement logico-mathématique :

$1023 \mathrm{Ma}$ : : parce que on peut faire soit/ heu l'autre y peut mettre soit treize ou soit quinze

1024 An. : non t'as dit

$1025 \mathrm{Ma}$. : heu quinze

La maîtresse tente de ramener ces derniers dans son projet didactique. Cependant, ses interventions sont toutes sous-tendues par sa logique: faire énoncer la liste "gagnante » :

-1011: // est-ce que vous croyez qu'il y a encore d'autres nombres qui sont importants/ avant encore ou bien/ c'est qu'à partir de onze que c'est intéressant

-1040 : moi j'connaissais le truc/j'suis arrivée

-1050 : allez essaie/ puisque tu penses que je sais pas jouer/ coquin/// vas-y

-1062 : hein/ elle gagne/ quelle coquine cette Anne-Marie// qu'est-ce tu en penses ça a l'air de marcher ton/ ton truc

-1070 : mais// il manque le dix-sept/ donc/ si tu ne mets pas ces trois chiffres tu perds// elle a l'air de dire que si/ il faut qu'on ait absolument onze quatorze et dixsept

Le résultat est un piétinement des élèves qui traduit un échec du passage d'un contexte (le «jeu apparent») à un autre (le «jeu symbolique», celui où des notions mathématiques peuvent être découvertes).

Appelés à plusieurs reprises « coquin » ou « coquine », les élèves qui s'inscrivent dans le jeu de la maîtresse ne sont pas interpellés, car évalués dans une connivence entre illusionnistes habiles qui ne met devant aucune bifurcation, saluée par un clin d'œil du maître à qui partage sa « mètis » (Détienne : ruse pédagogique) car l'objectif est dans le résultat immédiat, non dans le cheminement dans un nouveau contexte. Pas de 
déplacement vers une signification nouvelle mais immobilisation de l'élève dans la recherche de «trucs".

41 Ni interpellation ni passage du jeu apparent au jeu symbolique, d'un contexte de sens commun à un contexte mathématique, pas de «décontextualisation / recontextualisation ». L'observation de la séance sur un plan topogénétique montre que les places énonciatives restent fortement inégales, les élèves étant soit peu invités à s'expliquer, soit incités à le faire sans référence aux règles du jeu, ce qui mettrait en jeu les conditions de réussite, donc favoriserait le changement de contexte.

\subsubsection{L'interpellation, moteur du jeu symbolique}

Dans l'autre classe, dite " $M$ », il n'en va pas de même. L'atmosphère y paraît tempêtueuse, et l'interpellation, lorsqu'elle est observable, renvoie à des gestes professionnels langagiers identifiables mais fonctionnant sur le mode de la série et en correspondance stricte avec la nature des objets à enseigner.

Le fragment de protocole cité en ANNEXE 1 se situe après un moment d'action où l'enseignant a, en cohérence avec ses principes didactiques, refusé le débat, et après une première phase de formulation, où les élèves ont joué par équipes invitées à se concerter. Le temps de débat est un temps de langage intermédiaire, au sein d'une situation de mise à distance de l'action, donc de construction d'une signification. D'où la bifurcation potentielle: des avancées se manifestent, mais ne sont ni théorisées sur le moment à l'aide de pratiques langagières formelles, ni conceptuellement stabilisées. Plusieurs aspects sont à noter pour rendre compte des interpellations magistrales.

La manière dont l'enseignant utilise l'interpellation pour gérer les interactions est motrice dans l'avancée conceptuelle des élèves: elle ne les enferme pas dans le jeu apparent puisqu'elle ne cherche pas à leur faire dire la liste gagnante, dont il n'est pas question; au contraire, les interpellant sans arrêt sur les règles du jeu, elle les amène à la fois à une position d'énonciateur rendue pertinente par la référence à ces règles, et à un questionnement sur les conditions de réussite, autrement dit à passer du possible (provenant du contexte de l'action requise par le jeu apparent) au nécessaire (contexte mathématique virtuel). La différence d'activité langagière entre les deux enseignants est saisissante :ici, après une mise en situation, elle se partage entre interpellations incitant tel élève à s'expliquer (la phrase interrogative est largement dominante), rappel au règlement (227: « Attention! (...)/ si c'est vrai ce que vous dites vous aurez trois points (... )») et rappels à l'ordre. Mais ici, ceux-ci ne traduisent pas un problème de discipline renvoyant à la " vie psychique du pouvoir ». Ils prennent acte du caractère intermédiaire de la situation : à la différence de la classe « $G$ », où il y a peu de problèmes de gestion de la classe car les élèves sont toujours dans le faire et n'occupent donc qu'une seule position énonciative, ici, il y a souvent rappel à la discipline pour recadrer les deux positions engendrées par la situation didactique même : faire et dire, se référer à l'action effectuée ou à ses conditions, au contingent ou au nécessaire.

282: « M : Ah parce que on a vu tout à l'heure quelqu'un / d'ailleurs c'était marqué (elle se tourne vers le tableau)/ c'est votre équipe/ c'est votre équipe qui avait démontré qu'avec 14 on gagnait et qu'avec 17 aussi / (elle entoure les nombres) alors qu'est-ce qu'elle a fait Eléonore/ elle a oublié complètement ce que vous aviez dit tout à l'heure /d'accord ? Ruth ? Ah tu as fait exprès/ tu as fait exprès de ne pas mettre le 14 / alors tu as fait exprès de perdre?» . 

points importants: le passage possible $\rightarrow$ nécessaire d'une part, illustré par 282 alors qu'en 275-278, comme en 204-213, l'enseignant «traite » l'implicite de la consigne en opposant l'ordre de l'universel (275) à celui du contingent (278). Plusieurs élèves semblent avancer et ultérieurement (312, non reproduit), un élève utilisera "de toute façon » dans le même sens : «Parce que si tu mets 6 ou 7, si tu mets 6 je rajoute 2, si tu mets 7 je rajoute 1 , on arrive à 8 de toute façon, après ça va faire 11,14 ». Et d'autre part, l'apparition, d'un début de discours de l'anticipation hypothético-déductive (Aurore, 297), qui participe de l'esquisse de dé-/re-contextualisation, en direction de quelque chose qui n'est plus le jeu effectué, mais une représentation écrite et conventionnelle de ce qu'il a produit.

\subsection{L'interpellation comme genre de l'activité didactique : l'entrée dans la culture d'écrit au CP vue par Jacques Bernardin (ANNEXE 2)}

49 Les différences de formes que prend ici l'interpellation sont à la mesure de celles des savoirs en jeu : ni notions, ni savoir-faire, mais un socle de représentations indispensables au traitement de la nature linguistique du langage écrit -avec un palier supplémentaire dans la diffraction de l'interpellation, répartie sur une multitude d'actes trouvant leur source dans la création d'un contexte d'acculturation: l'on va noter des effets 
d'interpellation sans pouvoir les rattacher à autre chose qu'à la dynamique même de la situation progressivement intériorisée par les élèves -en tout cas, à aucun acte de langage ponctuel, direct ou indirect, mais à un genre professionnel étroitement lié à la nature des savoirs en jeu: on peut aller jusqu'à dire que ce sont les savoirs qui interpellent. Strictement cadré par eux, le geste se réduit à quelques récapitulations mettant en évidence des contradictions. Mais dans le cadre d'un système d'énonciation adressé à un collectif : l'interpellation est consubstantielle du processus même de construction d'une communauté discursive; elle embraye sur les aspects émotionnels de l'acculturation. L'importance du facteur «savoirs " se note à ceci que l'interpellation ne discrimine, ne « spectacularise » pas un sujet isolé, mais une communauté discursive en formation

Dès le titre de l'ouvrage, J. Bernardin montre clairement que cet apprentissage n'est pas de ceux qui s'épuisent dans des prescriptions techniques ${ }^{4}$. L'un des moments - clés de cette acculturation est celui où, en début Décembre, il s'agit de savoir comment mettre de l'ordre dans un fichier regroupant les mots identifiés depuis le début de l'année. Le débat sur la méthode de classement va être celui de la bascule d'une vision logographique des mots à une vision linguistique, passant par la perception de la nature alphabétique du langage écrit. Or, toutes les propositions des élèves ne s'appuient pendant un temps long, que sur le sens: on va mettre « bateau » avec « eau »... « parce que le bateau va dans la mer ». D'où l'échec: un même mot va se retrouver dans plusieurs catégories, un autre dans aucune -jusqu'au moment où s'enclenche, comme par miracle, le moment qui va progressivement- tout débloquer :

Florian : Moi, je propose « chasse » avec « cheval », « chien » parce que des fois, il y

a des chasseurs qui ont des chiens...

(J'affiche.)

Sandy : C'est pareil...

Mélodie : C'est presque pareil..

$\mathrm{M}:$ Qu'est-ce qui est pareil ?...

Mélanie : La première lettre...

Sandy : On peut mettre « jour » avec « jouer » parce que ça se ressemble...

Florian : Ça se ressemble pas trop...

Mélanie : Si, les premières lettres ! Et aussi « jouet », ça se ressemble...

51 N'y aurait-il dans cette trouvaille providentielle, ouvrant la voie à l'apprentissage de la nature alphabétique du système d'écriture français, qu'un de ces coups du hasard sauvant bien des enseignants de situations didactiques hasardeuses ? Plusieurs éléments permettent de supposer une tout autre origine :

* à court terme, la posture du maitre par rapport au « jeu » institué est assez semblable à celle de la maîtresse de la classe « $\mathrm{M}$ » dans le cas de la course à 20 . Son usage du principe de non-contradiction est son arme essentielle; il ne cherche pas à focaliser sur les résultats partiels possibles (par exemple, deux élèves ont rapproché " tête » de "fête ", sans entraîner de réaction de sa part sur la position des lettres semblables) : il rappelle simplement la règle, et montre le paysage contradictoire à arpenter :

«Vous voyez, il y a plusieurs problèmes: on ne sait plus si «bois» va avec

" chasse " ou avec " ours ", " cheval» et " chien »; vous me dites, ici, ce sont les

animaux, et en même temps, « souris ", vous le mettez avec « eau » et « chasse... »

* à plus long terme, cette séance reçoit les effets d'une série d'autres séances centrées sur les propriétés du système d'écriture alphabétique à travers le classement d'écritures allant des peintures rupestres aux tablettes de cire romaine, en passant par idéogrammes, cunéiforme, etc. Les élèves parviennent à trouver que les tablettes romaines présentent le 
système d'écriture le plus proche du nôtre, et le professeur procède alors à un apport de connaissances techniques sur la question, mais dans son analyse, il tient à faire remarquer que l'essentiel est peut-être ailleurs. En effet, cette découverte met en jeu des émotions :

«Ce qui a été frappant, c'est l'implication très forte des enfants (il a fallu être ferme pour organiser la prise de parole, ce qu'ils avaient à dire ne supportait pas d'attendre!) et -en contraste apparent- la qualité d'écoute et d'attention lors de l'intervention magistrale qui venait valider leur travail. Les exclamations spontanées laissaient penser qu'au delà de l'apport de connaissances historiques, ce dont il s'agissait, c'était d'abord d'émotion. Sentiment d'inscription dans une histoire humaine qui nous dépasse et nous fascine, émotion fondamentale du questionnement des origines. » (p. 77).

On ne saurait mieux illustrer l'ouverture de sens propre à une véritable posture d'arpenteurs : les élèves vont essayer leur trouvaille sur tous les mots pour construire le principe alphabétique, avec profusion de traces d'un réel processus de subjectivation, et d'indices autorisant sur le moyen ou le long terme l'hypothèse que du développement est en cours. L'interpellation investit le sujet.

\section{Conclusion : l'école, l'interpellation, l'humanisation}

Quelques remarques pour ouvrir divers champs de réflexion.

On ne peut confronter approche linguistique et approche didactique d'un phénomène si le langage n'est pas considéré comme une activité et analysé comme tel, dans la mesure où à la fois il réalise un certain rapport du sujet au monde et au contexte, et où il constitue la matrice de ce rapport. Il est évidemment impossible d'instruire ici cette question ${ }^{5}$.

D'autre part, l'interpellation déborde de sa fonction idéologique, à laquelle une approche trop transversale, insuffisamment attentive à la question des savoirs et de leur spécificité, risque de réduire l'école. Certes, tout contrat comporte une fixation, mais celle-ci participe de la subjectivation de plusieurs manières différentes, selon qu'elle ne renvoie qu'à des identités préconstruites ou qu'elle joue un rôle de tremplin, qu'elle permet l'adhésion à des fonctions réorganisatrices, que sa mise en situation permette la construction d'outils psychologiques. La description et l'analyse de la signification de l'interpellation exigent donc un cadre plus large que l'acte d'énonciation ou le "geste » professionnel : c'est au genre de l'activité, au genre professionnel qu'il faut se référer pour saisir ce qui peut être ouvrant à partir d'une fermeture : naviguer entre les deux dimensions est une condition de l'avancée du temps didactique et du développement de la topogenèse. Les variations imputables à celles de la nature des objets de savoir ne font que renforcer cette exigence. Les effets contradictoires de l'interpellation, qui ne dépendent parfois d'aucun acte, d'aucun geste, peuvent être identifiés au sein de la propriété dynamique des situations à construire un contexte intra-psychologique. L'approche didactique serait la seule à pouvoir articuler un propos cohérent sur la question, à condition d'intégrer la dimension psychologique de l'apprentissage, exigeant une méthodologie adéquate : la question est de savoir comment l'élève peut se sentir mis en mouvement. A considérer ce qui fait la substance des interactions langagières, le développement des capacités psychiques de l'élève ne peut venir que du sentiment 
pratique d'un accès possible aux " secrets des adultes », comme l'écrivait Bettelheim. Dans ce cadre, l'interpellation est ouvrante, incite à « arpenter »...

L'intérêt de son analyse dialogique est au fond là : nous aider à tracer une ligne de démarcation entre savoirs, situations et normes inscrits dans une instrumentation portant en elle-même son propre système de références, et savoirs, situations et normes inscrits sur un horizon d'humanisation : en font partie les fonctions symboliques sous les espèces des lois des nombres ou de "l'algèbre du langage" (Vygotski), qui ne se construisent pas comme coule un long fleuve tranquille... La Zone de Développement Prochain est un espace de rififi, qui n'avance pas sans bifurcations provoquées du sujet, sans effets d'interpellation. Prise entre cette nécessité et celle que lui vaut sa nature spécifique d'institution, l'école n'a pas fini de se chercher en soumettant ses fonctionnements, ses contenus, ses disciplines, ses ingénieries, l'épistémologie pratique de ses professeurs, à l'alternative qui constitue l'essence même de la question de l'interpellation.

\section{BIBLIOGRAPHIE}

Bernardin J. (1997). Comment les enfants entrent dans la culture écrite, Paris : Retz.

Bernié J.-P. (1998). Eléments théoriques pour une didactique interactionniste de la langue maternelle, in Apprendre à l'école - Perspectives Piagétiennes et Vygotskiennes, M. Brossard \& J. Fijalkow éds., Bordeaux : PUB.

Bernié J.-P. (2001). Genres discursifs scolaires, genres de l'activité et conceptualisation, in Apprentissage, développement et significations, J.-P. Bernié dir., Bordeaux : PUB.

Bernié J.-P. (2002). L'approche des pratiques langagières scolaires à travers la notion de " communauté discursive " : un apport à la didactique comparée ?, Revue Française de Pédagogie, $\mathrm{n}^{\circ}$ 141, Paris : INRP.

Bernié J.-P. (2005). Les « situations différées » de Francis Ruellan : fécondité et zones d'ombre d'un espace de problématisation, in Pédagogie du projet et didactique du français - Penser et débattre avec Francis Ruellan, Y. Reuter dir., Lille, Editions du Septentrion.

Butler J. (1997). The Psychic Life of Power - Theories in subjection, Palo Alto : Stanford University Press.

Grize J.-B. \& Piéraut-Le Bonniec G. (1983). La contradiction - Essai sur les opérations de la pensée, Paris : PUF.

Grossen M. (2001). La notion de contexte : quelle définition, pour quelle psychologie ?, In Apprentissage, Développement et significations, J.-P. Bernié dir., Bordeaux : PUB.

Jaubert M. (2007). Langage et construction des connaissances à l'école, Bordeaux : PUB.

Jorro A. (2001). L'évaluation comme ouverture du sens, L'activité évaluative réinterrogée Regards scolaires et socioprofessionnels, G. Figari \& M. Achouche dir., Bruxelles : De Boeck. 
Martin R. (1987). Langage et croyance - Les « univers de croyance » dans la théorie sémantique, Bruxelles : Mardaga.

Maulini O. (2005). Questionner pour enseigner \& pour apprendre : Le rapport au savoir dans la classe. Paris : ESF.

Ong W.-J. (1982). Orality and Literacy : The Technologizing of the Word, London and New York : Routledge.

Rebière M. (2001). Une notion venue d'ailleurs... la posture, In Apprentissage, Développement et significations, J.-P. Bernié dir., Bordeaux : PUB.

Ruellan F. (1999). Un mode de travail didactique pour l'enseignement : Apprentissage de l'écriture au Cycle 3 de l'Ecole Primaire, Thèse pour le doctorat en Sciences de l'Education, Y. Reuter dir., Université Charles de Gaulle - Lille III

\section{ANNEXES}

\section{Annexe 1. Extrait de la « Course à $20 \mathrm{M}$ »}

$195 \mathrm{M}$ : Asseyez-vous/ Je crois que là dans chaque équipe vous avez découvert des choses permettant de gagner à coup sûr/j'ai bien vu à vos têtes /Eh bien maintenant/ vous allez proposer ce que vous avez découvert/ un par groupe/ si ce que vous proposez (elle lève les bras et dit): non/ on va faire pareil/ de la même manière/ si ce que vous proposez est juste et que vous avez réussi à le démontrer parce qu'il ne suffit pas de dire/ c'est comme cela/ il va falloir ou l'essayer ou trouver un moyen de prouver que ce que vous dites/ c'est vrai Si ce que vous avancez est vrai/ vous marquerez un point/ si par contre ce que vous proposez l'autre équipe arrive à démontrer que c'est pas vrai/ hein/ ce que vous proposez c'est pas vrai/ l'autre équipe gagnera 3 points

196 On entend oh la la

$197 \mathrm{M}:$ d'accord?

198 M à Denise : je vais peut-être pas faire comme cela ... allez parce que (xxx)

199 M Allez / Alors qui c'est qui qui vient de jouer là ? qui a démarré ? (M montre la partie encore au tableau)

200 Des élèves disent: B

$201 \mathrm{M}$. alors l'équipe A on va leur demander leur première découverte

202 Dieudonné/ tu viens au tableau/ pour un point / il va nous proposer une découverte qui d'après lui permet de gagner à coup sûr / C'est quoi ? M efface le tableau

$203 \mathrm{D}$ si on met le 14/ on est obligé de mettre le 17/avec le 17 on est obligé de gagner

204M alors/ est-ce que tout le monde ?(nefinitpas)

$205 \mathrm{M}$ écrit au tableau : si on met 141 après qu'est

ce que tu as dit?

206D : si on met le 14/ alors l'adversaire il est

obligé de mettre 16/ il met 15 ou 16/ 
207M continue d'écrire Mécrit au tableau au fur et à mesure

$208 \mathrm{D}$ : et comme ça marche en 1 ou en $2 /$ on peut

mettre 17/

$209 \mathrm{M}$ alors/ on peut ou il faut? A B

$210 \mathrm{D}$ il faut mettre 17 Si on met $14 \rightarrow 15$

211 On entend un élève de l'autre groupe qui 16

répond en même temps 17->18

212 M (à cet élève mais attends/ attention je te 2019

signale que c'est eux qui gagnent le point/ donc

si tu les aides/ hé bien tant mieux pour eux/ tant

mieux pour eux

213 D : il faut mettre IV Et l'adversaire si il met/ si

il met/ si moi/ je mets 17/ l'adversaire il est

obligé de mettre 18 ou 19/ et comme ça marche

en 1 ou en 2/ comme je l'ai déjà dit/ on met 20

$214 \mathrm{M}$ est-ce que tout le monde est convaincu de ce qu'a trouvé/ un point/ un point pour l'équipe A

215 M l'équipe B/ l'équipe B / Non mais dis donc/ Philibert /allez hop

$216 \mathrm{E}(\mathrm{xxx})$

$\mathrm{M}$ : ah tu es déjà venu/ toi ? Alors non/ Safia /allez

$\mathrm{E}:(\mathrm{xxx})$

M / allez stop Safia/ hé oui/ vous êtes une équipe/ alors n'importe qui dans l'équipe peut être appelé bien sûr

E : on peut l'aider?

$\mathrm{M}$ : Ah non / vous pouvez pas l'aider là

$\mathrm{F}(\mathrm{S}$ ?) : juste avant on sait qu'on peut faire un truc

M : vas-y

F S ?) : juste avant on peut mettre on peut mettre 11. Elle écrit 11 au tableau

$\mathrm{M}: 11$ / si on met $11 /$ on gagne?

E : oui

$\mathrm{M}$ : alors/ si tu le prouves ? S'adressant à quelqu'un de la classe : attention/ après vous aurez la parole/ si c'est pas bon vous aurez la parole/ si c'est vrai ce que vous dites vous aurez trois points/ là on joue pour un point.

Safia dessine une flèche après 11 et dit : il met soit 1 soit 2

M reprend la craie et écrit 11 圈 12 ou 13 / s'adresse aux B qui parlent : moi/ je vais vous enlever le point/ c'est elle qui parle 
M : alors/ il met 12 ou 13/ d'accord/

Safia : (xxx) elle écrit un 2 après le 13.

M efface ce 2/ dit : quelle équipe ? ajoute un B au dessus du 11 un A au dessus du 12 / S'il met $12 /$ tu mets quoi/ toi?

Safia : je mets 14. M écrit 14

M : Et s'il met 13?

Fa : je mets 14

M (en écrivant 14 dans la colonne B): elle met 14 /alors?

Safia : parce que (xxx)

$\mathrm{M}$ : alors/ est-ce que vous êtes d'accord ? La bas? $\mathrm{p}$

Eléonore : Non parce que moi/ à un moment j'avais joué / j'avais mis 12 après j'ai mis 14 après il a mis 15/ après j'ai mi 17

E : c'est pas forcé / on peut changer ! Brouhaha (xxx)

$\mathrm{M}: \mathrm{ah}$ : stop / stop Eléonore/ tu as entendu ce qu'ont dit tes camarades de ta proposition/ et qu'est-ce que t'en penses?

Eléonore : $(\mathrm{xxx})$

Brouhaha. Plusieurs enfants parlent en même temps.

$\mathrm{M}$ : qu'est-ce que t'en penses ? On ne gagne pas tout le temps avec 11 ?

$\mathrm{E}: \mathrm{Si}$

$\mathrm{M}$ : bon / alors on joue/ on joue/ Eléonore tu viens jouer contre Safia/ puisque Safia dit qu'on gagne tout le temps si on met 11/ D'accord/ alors une là/ l'autre là./ et attention / là c'est pour 3 points/ attention attention là c'était pour un point/ là c'est pour 3 points/ vous étiez pas d'accord / ça veut dire que si vous n'avez pas raison/ c'est les autres qui remportent ces 3 points. Hé oui / On y va/ attention on y va.

Safia s'adresse à Eléonore : vas-y commence

Eléonore : non à toi

$\mathrm{M}$ : alors Safia/ commence

Safia écrit 1 Eléonore: 2 puis à tour de rôle : 3578911 Les 2 filles jouent très lentement.

Safia écrit 12/ Eléonore réfléchit...

$\mathrm{M}$ : allez Eléonore

Eléonore écrit 13/ aussitôt Safia saute de joie et écrit 14

Cris de joie de l'équipe $B$

E de l'équipe A : Bravo/ maintenant on a perdu!

Les 2 filles continuent à jouer pendant que $M$ essaie désespérément de calmer les autres

M : Alors / alors /bon/ on fait le point là/

Kamal dit qu'on n'est pas obligé de gagner avec 11 et qu'est-ce qu'il dit Thomas? 
$\mathrm{E}:(\mathrm{xxx})$

$\mathrm{M}$ : Thomas/ Thomas il sait jouer avec 11/ Kamal il a dit qu'on gagne pas à tous les coups / Vous venez tous les 2

Pendant que les enfants discutent avec animation/M efface le tableau/les 2 garçons arrivent

Alors Thomas a dit qu'il sait qu'il est sûr de gagner s'il met 11/alors Thomas met 11/ M écrit 11 et on y va

Thomas : vas-y

Kamal : il part de 11, s'il joue avec il va gagner.

Thomas : Hé bien voilà !

M : ah ben/ le but/ c'est quoi ?/ c'est de savoir jouer M (à la classe) : Attention/ attention / Kamal/

$\mathrm{E}:(\mathrm{xxx})$

$\mathrm{M}$ : ils ont raison alors ? 11 ça permet de gagner/ alors pourquoi vous contestez si $(\mathrm{xxx})$ ?

Thomas : hé oui / Tout le monde parle à la fois et crie

Denise : non/ non/

M : Ruth /bon /Ruth /Ruth /alors pourquoi tu n'es pas contente et pourquoi tu dis qu'il triche? on t'écoute

Ruth : parce que $(\mathrm{xxx})$ Eléonore elle a perdu

M : alors pourquoi elle a perdu ? Pourquoi elle a perdu ? Pourquoi elle a perdu ? Eléonore, est-ce que tu peux dire pourquoi tu as perdu?

$\mathrm{E}:(\mathrm{xxx})$

$\mathrm{M}$ : Stop / pourquoi elle a perdu?

Eléonore : $(\mathrm{xxx})$ ?? ?

$\mathrm{E}$ : hé voilà/ hé voilà/

$\mathrm{M}$ : Non tu n'as pas perdu parce que tu as perdu/ non/ Eléonore est-ce que tu sais pourquoi tu as perdu tout à l'heure?

E : oui / oui

M : alors que tu avais mis 11 ? Alors c'est pourquoi?

$\mathrm{E}:(\mathrm{xxx})$

$\mathrm{M}$ : Ah parce que on a vu tout à l'heure quelqu'un / d'ailleurs c'était marqué (elle se tourne vers le tableau)/ c'est votre équipe/ c'est votre équipe qui avait démontré qu'avec 14 on gagnait et qu'avec 17 aussi / (elle entoure les nombres) alors qu'est-ce qu'elle a fait Eléonore/ elle a oublié complètement ce que vous aviez dit tout à l'heure /d'accord? Ruth? Ah tu as fait exprès/ tu as fait exprès de ne pas mettre le 14 / alors tu as fait exprès de perdre?

Denise : tu as fait exprès de leur donner 3 points?

$\mathrm{E}:(\mathrm{xxx})$ 
$\mathrm{M}$ : Eléonore

$\mathrm{E}:(\mathrm{xxx})$

$\mathrm{M}$ : elle veut prouver que quoi?

$\mathrm{E}:(\mathrm{xxx})$

$\mathrm{M}$ : hé bien si quand on sait jouer on est obligé de gagner/ donc vous leur avez donné 3 points

Brouhaha général

Je reprends/ stop/je reprends/ j'attends vraiment que vous vous calmiez / c'est n'importe quoi/ non je n'entends plus rien/ c'est moi qui parle / L'équipe B a dit avec 11/ on gagne/ ici vous avez dit avec 11 on perd/

Remous du côté de l'équipe A

E : pas obligé

M (montrant le tableau): hé bien / la preuve / Kamal/ On sait jouer/ on sait jouer/ le but c'est qu'on sait jouer!

$\mathrm{E}:(\mathrm{xxx})$

$\mathrm{M}$ : non mais ils savent jouer ! / tu dis n'importe quoi Kamal/ tu dis n'importe quoi/ Kamal/ Attention/ on continue/ qui a trouvé d'autres découvertes ... Aurore

Aurore : je dis que si on met 8 on gagne parce que l'autre va mettre 9 ou 10 et si après on met 9 on rajoute 2 ça fait 11 / si on met 10/ on rajoute 1 et ça fait 11 / et après on peut continuer jusqu'à 20 (elle montre la suite au tableau).

\section{Annexe 2. Jacques Bernardin, « Comment les enfants entrent dans la culture} écrite ", Retz 1997, pp. 103-105.

$\mathrm{M}: \mathrm{D}$ 'autres idées?

Émilie : «fête » avec « tête »... (Moment d'arrêt interrogatif dans la classe.)

$\mathrm{M}$ : Vous voyez pourquoi elle propose cela?

(Plusieurs) Oui!...

$\mathrm{M}$ : Pourquoi?

Emilie : Parce que ça ressemble...

Florian : Non, il y a une lettre (dans « fête ») qui est pareille que dans « tête », le « e » avec un accent...

(Plusieurs) «... Ête »...

Florian :... Mais dans 9 « tête », il y a un « $t$ » au début.

$\mathrm{M}:$ On continue...

Mélanie : On pourrait mettre « bateau » et « eau »... parce que le bateau va dans la mer...

(Plusieurs) ... Avec «mer» aussi!

Mélodie : «Lit» avec «tête»... parce qu'on met la tête sur le lit ... 
M: Je vous signale qu'on avait mis « tête » avec « chapeau », avec « école " et « ami » ...

Mélodie : «Voiture» avec «ville»... parce que les voitures sont dans la ville.

Mélanie : On pourrait mettre « lait » avec tout ce qui se mange... et «fenêtre» avec «maison»...

Gwendoline : «Mer» avec «bateau»... parce que les bateaux vont sur l'eau...

Mélodie : «Voir» avec «regarder»... parce que ça veut dire presque la même chose...

Sandy : « Chasse » avec « bois » parce qu'on va à la chasse dans les bois...

Florian : Oh, la, la!... Ben et l'«eau»?...

$\mathrm{M}$ : Vous voyez, il y a plusieurs problèmes : on ne sait plus si «bois » va avec « chasse » ou avec « ours », « cheval » et « chien »; vous me dites, ici, ce sont les animaux, et en même temps, « souris », vous le mettez avec « eau » et « chasse »...

Mélodie : On met « souris » avec "bois" "chasse" «cheval» et « chien »...

Florian : Eh ben non! Parce que la souris, ça vit pas dans le bois... Alors, il faut changer, il faut trouver autre chose : "fête" on n'a pas réussi à le ranger; « eau », on ne sait pas si c'est avec « bateau » et « mer » ou si c'est avec «feuille »; " chasse», on ne sait pas où le mettre... Ça ne va pas vraiment bien, notre classement.

M:On n'arrive pas à classer toutes les fiches d'une part, et des fiches se promènent entre différentes «familles » d'autre part, ça ne va pas...

Florian : Moi, je propose « chasse » avec « cheval », « chien » parce que des fois, il y a des chasseurs qui ont des chiens...

(J'affiche. )

Sandy : C'est pareil...

Mélodie : C'est presque pareil...

M : Qu'est-ce qui est pareil?...

Mélanie : La première lettre...

Sandy : On peut mettre «jour» avec «jouer» parce que ça se ressemble...

Florian : Ça se ressemble pas trop...

Mélanie : Si, les premières lettres! Et aussi «jouet», ça se ressemble.. *

Gwendoline : Bébé mange un bout de pain... (Les autres) : Non!...

$\mathrm{M}$ : Sandy, lu veux bien expliquer pourquoi tu avais proposé de mettre ces mots ensemble ? Montre précisément.

Sandy : Parce que c'est pareil...

(Corinne vient montrer plus précisément ce qui est vraiment semblable).

Mélanie : « Fenêtre » et « fête »... (montre ce qui est pareil)... et aussi « fille » et « feuille ».

Florian :... Et « enfant » aussi.

(Les autres) Non... C'est pas au début!

Aurélien : «Ville » et « voiture » ... (montre ce qu'il trouve semblable) ... 
Gwendoline : ... Avec « vélo » ...

Mélodie : Et «voir»... Au début, on a les mêmes... Corinne (4) «Maman», «main»...

Aurélien : On peut mettre «manger» aussi...

Sandy (excitée) : On peut mettre «chien» avec «chambre»! Le début, c'est le même!...

Aurélien : Y a même « chapeau »!

Mélanie : On pourrait mettre aussi «jardin » avec «jour » ... c'est le même début.

Corinne : Là, il y a « soupe » et « souris »...

Florian : Ce qui serait bien, c'est « regarder » et « rue » ...

$\mathrm{M}$ : Oui, pourquoi?

Florian : Parce que « $\mathrm{r} », \ll \mathrm{r} » \ldots$

Sandy : « Bateau » avec « boule »... avec « bois » et « bébé »...

$\mathrm{M}:$ Tu veux bien mettre le nom de la famille?

J'entoure les fiches du tableau et accroche à l'ensemble ainsi constitué une « étiquette » avec la craie... (Plusieurs) : «B»! Mélanie : C'est comme l'alphabet.

Alice : «École» et «étoile»... parce qu'il y a un « e » avec un accent.

Aurélien : «Lait», «lettre», «lit»...

$\mathrm{M}$ : Montre-nous ce qui te fait dire que c'est une même famille...

(Montre le «l"). Mélodie :... Et « lune »! (Les autres) Oui!... (Aurélien (7) complète en écrivant le «nom» de la famille : "l" ce qui sera le cas désormais pour chaque famille constituée ... )

Florian : Moi, je suis pas du tout d'accord sur la famille des « 1 », parce qu'à « lait », il y a un « $a » .$.

$\mathrm{M}$ : Attention, qu'est-ce qu'on a choisi de faire?..

Mélanie : La première lettre, elle est bonne... (Les autres) Oui.

$\mathrm{M}$ : On peut faire d'autres familles?

Alice : « Montre » et « mer »... avec « maison », « main », « maman », « manger »... (« $m$ »)

Mélanie : ... C'est moi qui l'avais dit!...

(Il y avait doute sur l'indice discriminant : était-ce « $\mathrm{m} » \mathrm{ou} « \mathrm{ma} » ? .$.$) .$

\section{NOTES}

1. Acte I, sc. 3 : « « All hail, Macbeth! Hail to thee thane of Glamys !

All hail, Macbeth! Hail to thee thane of Cawdor!

All hail, Macbeth! that shalt be king hereafter !"

2. C'est en effet l'intonation qui permet de faire la différence entre remarques ou questions « ordinaires » (Maulini, 2005), et interpellation. Dans une autre séance où il faut retrouver sur le tableau les questions pertinentes pour résoudre un problème, la transcription peut faire disparaître la vraie nature de la question posée par le maître : « Où sont-elles, ces questions ? ». Si 
un accent d'intensité est mis sur « elles ", il s'agit d'une simple demande d'information, mais s'il porte sur « où », l'on voit l'élève interpellé sur un aspect-clé du problème : retrouver l'endroit permet de différencier ces questions et d'autres éléments du cours, c'est peut-être aussi mobiliser les souvenirs d'un débat antérieur sur la question : enjeu de pertinence épistémique. D'où notre position sur l'aspect intonatif plus que lexicalement ou syntaxiquement marqué de l'énonciation interpellative.

3. Association pour des Recherches Comparatistes en Didactique.

4. Contrairement à ce que voulurent imposer le ministre De Robien et ses sbires en opposant, en 2006, le « syllabique » au fantasme du « global »...

5. On se réfère aux perspectives Bakhtiniennes et Vygotskiennes, malgré leurs différences philosophiques, à l'oeuvre de Léontiev, aux travaux de Bronckart, d'Yves Clot, de Daniel Faïta et de leurs équipes, ainsi qu'aux contributions de la nôtre.

\section{RÉSUMÉS}

L'objectif est de montrer que l'interpellation, loin de se réduire à un acte monologique ou un " geste », constitue un genre de l'activité didactique, dans le cadre, non d'une énonciation isolée mais d'une co-activité foncièrement dialogique. Vue sous cet angle, elle présente diverses propriétés, notamment l'indétermination des effets, qui ne permettent pas de l'analyser sans prise en compte des objets de savoir en jeu, et sans examen des situations et des contextes. Sous cet angle, elle se révèle un site précieux pour observer la réorganisation des capacités psychiques supérieures sous l'effet de l'acquisition des savoirs, en particulier parce qu'elle met en lumière les bifurcations qui caractérisent les parcours d'apprentissage. Etudier l'interpellation exige donc un élargissement des unités observées mais aussi une transformation de l'approche du langage, dans les termes de la théorie de l'activité.

This paper aims to show that interpellation cannot be reduced to monological acts or gestures, but makes up a didactic activity genre, within the framework of a co-activity basically dialogical. From this point of view, it affords various properties, particularly an indecisiveness of effects, which doesn't allow any analysis excluding specific knowledges at stake, or without examination of situations or contexts. Seen from that angle, it makes up a precious observatory of the reorganization of higher psychical abilities under the effects of knowledge acquiring particularly because it brings to light the forks proper to the apprenticeship courses. So, the analysis of the interpellation requires the widening of the observed units, and a new approach of language, as shown in the activity theory.

\section{INDEX}

Mots-clés : co-activité, dialogisme, contexte, topogenèse, fictionnalisation

Keywords : co-activity, dialogism, context, topogenesis, fictionalization 
AUTEUR

JEAN-PAUL BERNIÉ

IUFM d'Aquitaine 